\title{
Editorial
}

\section{Patient flows: runners, specials and strangers}

The Glenday Sieve is an approach used in the healthcare setting to identify certain groups of procedures, conditions or activities that share common elements (1). Grouping them on the basis of volumes of activity serves to identify conditions for building cost-effectiveness improvement strategies. Furthermore, by focusing on a few high-volume activities, constituting a hospital's main activities, it is possible to identify small goals that are effective for improving the "patient's journey". In this regard, the British National Health System (NHS) has analyzed patient flows, dividing them into three groups corresponding to three patient categories: runners, characterized by high volumes of activity and a pathway that is rapid, standardized, highly predictable and, in more than $90 \%$ of cases, planned; specials, characterized by high volumes of activity and a personalized, partly standardized (albeit with the possibility of additional phases) and plannable (although not always) pathway; and strangers, characterized by low volumes of activity, unique requirements, unpredictable patterns of demand, and potentially long and complex pathways (2). A particularly interesting aspect of these patient flows is that there is no correspondence between volumes of activity and procedures analyzed in quantity terms. In other words, the bulk of the activity refers to a small number of procedures.

The Glenday Sieve is based on the Pareto Principle, which is used to group and separate procedures, conditions and activities on the basis of current volumes and is normally able to identify several categories that can be applied to any hospital, department or service. Categories standardized on the basis of observed and estimated volumes are normally assigned the following codes: green (corresponding to $50 \%$ of volume of activity and $6 \%$ of procedures), yellow (45\% of volume of activity and $44 \%$ of procedures), blue (4\% of volume of activity and $20 \%$ of procedures) and red (1\% of volume of activity and $30 \%$ of procedures).

This grouping system produces rather surprising results, and many health management professionals still have difficulty working out how a small percentage of procedures can represent around 50\% of their organization's volume of activity. Hip and knee replacement surgery are two operations that have been recognized as high-volume procedures. Patients undergoing these procedures must thus be considered runners; furthermore, given the significant levels of activity involved, it is important to underline that optimizing the pre-, peri- and postoperative course of these patients could have significant repercussions in terms of the use of human and material resources. In a similar way, prevention of possible intra- or postoperative complications could allow a significant reduction of the overall costs, direct and indirect, of a hospital or of an entire health system.

With this in mind, I wish to draw your attention, in particular, to two of the articles published in this issue of Joints. The first by $F$. Boniforti, presents an economic analysis, relating to a single hospital, of the direct and indirect costs of hip and knee replacement surgery. The second, by G. Solarino et al., looks at strategies for preventing infections in joint replacement surgery, focusing on the preoperative period.

Giuseppe Milano, MD Editor-in-Chief, Joints

\section{References}

1. Glenday Sieve - Runners, Repeaters, Strangers. Available at: http://www.institute.nhs.uk/quality_and_service_improvement_tools/quality_and_service_improvement_tools/glenday_sieve__runners_repeaters_strangers.html. Last access: December 3, 2015.

2. Understanding the Patient Journey. Process Mapping and Value Stream / Flow Analysis. Available at: http://www.qihub.scot.nhs.uk/media/162308/patient_journey.ppt. Last access: December 3, 2015. 